\title{
Enhanced liver progenitor cell survival and differentiation in vivo by spheroid implantation in a vascularized tissue engineering chamber
}

Kiryu K. Yap ${ }^{1}$, Aaron M. Dingle ${ }^{1}$, Jason A. Palmer ${ }^{1,3}$, Raminder Dhillon ${ }^{1}$, Zerina Lokmic ${ }^{1,2,5}$, Anthony J. Penington ${ }^{1,2,5}$, George C. Yeoh ${ }^{4}$, Wayne A. Morrison ${ }^{1,2,3}$,

Geraldine M. Mitchell $1^{1,2,3 *}$

Author Institutional Affiliations:

1. O'Brien Institute, 42 Fitzroy Street, Fitzroy, 3065, Melbourne, Victoria, Australia

2. University of Melbourne Department of Surgery at St Vincent's Hospital, Fitzroy, Melbourne, Victoria, 3065, Australia

3. Faculty of Health Sciences, Australian Catholic University, Fitzroy, Melbourne, Victoria, 3065, Australia

4. Centre for Medical Research, Western Australian Institute for Medical Research, and School of Chemistry \& Biochemistry, University of Western Australia, Perth, Australia

5. Murdoch Childrens Research Institute, Parkville, Victoria, Australia

Author Postal addresses:

Kiryu Yap: O’Brien Institute, 42 Fitzroy Street, Fitzroy, Melbourne, Victoria, 3065, Australia

Aaron Dingle: O’Brien Institute, 42 Fitzroy Street, Fitzroy, Melbourne, Victoria, 3065, Australia

Jason Palmer: O’Brien Institute, 42 Fitzroy Street, Fitzroy, Melbourne, Victoria, 3065, Australia

Raminder Dhillon: O’Brien Institute, 42 Fitzroy Street, Fitzroy, Melbourne, Victoria, 3065, Australia 
Zerina Lokmic: Murdoch Childrens Research Institute, Royal Children's Hospital

Flemington Road, Parkville, Victoria 3052 Australia

Anthony Penington: Murdoch Childrens Research Institute, Royal Children's Hospital

Flemington Road, Parkville, Victoria 3052 Australia

George Yeoh: Centre for Medical Research, Western Australian Institute for Medical Research, and School of Chemistry \& Biochemistry, University of Western Australia, Nedlands, Perth, Western Australia, 6009, Australia

Wayne Morrison: O’Brien Institute, 42 Fitzroy Street, Fitzroy, Melbourne, Victoria, 3065, Australia

*Corresponding Author: Associate Professor Geraldine Mitchell (PhD)

O’Brien Institute

42 Fitzroy Street, Fitzroy, 3065, Victoria, Australia

e-mail: geraldine.mitchell@svhm.org.au

telephone: +6139288 4030: fax: +61394160926

Running Headline: Spheroid-based tissue engineering with liver progenitor cells 


\section{ABSTRACT}

Liver tissue engineering is hampered by poor implanted cell survival due to inadequate vascularization and cell-cell/cell-matrix interactions. Here, we use liver progenitor cell (LPC) spheroids to enhance cell-cell/cell-matrix interactions, with implantation into an angiogenic in vivo mouse chamber. Spheroids were generated in vitro in methylcellulose medium. Day 2 spheroids were optimal for implantation $(22,407+/-645$ cells/spheroid $)$, demonstrating maximal proliferation (Ki67 immunolabeling) and minimal apoptosis (caspase-3 immunolabelling). In vivo chambers established bilaterally on epigastric vessels of immunodeficient mice were implanted with equivalent numbers of LPCs as a cell suspension (200,000 cells), or spheroids (9 spheroids). At day 14, a trend of increased LPC survival was observed in spheroid-implanted chambers [pan-cytokeratin (panCK + ) cells, $p=0.38,2.4$ fold increase)], with significantly increased differentiation [cytokeratin $18(\mathrm{CK} 18+)$ cells, $p<0.002$, 5.1 fold increase)] compared to cell suspension-implanted chambers. At day 45, both measures were significantly increased in spheroid-implanted chambers (panCK, $p<0.006,16$ fold increase) (CK18, $p<0.019,6$ fold increase). Hepatic acini/plates of CK18+ cells expressed hepatocyte nuclear factor 4- $\alpha$ and $\beta$-catenin, indicating ongoing hepatic differentiation. Spheroid cell-delivery significantly increased LPC survival and differentiation compared to conventional cell suspensions. This LPC spheroid/vascularized chamber model has clinical potential to generate three-dimensional vascularized liver tissue for liver replacement.

Key words: liver progenitor cells; multicellular spheroid; single cell suspension; vascularized in vivo tissue engineering chamber; implanted cell survival and differentiation; liver regeneration 


\section{INTRODUCTION}

Cell-based therapies offer an alternative to organ transplantation for use in the treatment of liver failure. However, despite attempts to directly transplant heaptocytes into various locations including the portal vein, liver parenchyma, and spleen [1], a significant threedimensional mass of liver tissue often does not result due to poor cell engraftment and expansion. This is likely due to inadequate vascularization at the implantation site and lack of appropriate cell/cell and cell/matrix interactions $[2,3]$. Cell retention may be enhanced by tissue engineering techniques where cells are combined with a scaffold to facilitate cellmatrix interactions, and implantation into a specific site such as the kidney capsule [4] or muscle [5] which constrains transplanted cells to the region. Nevertheless, the need for a rapidly developing blood supply remains an important obstacle.

The mouse vascularized chamber model used in this study has been developed to address vascularization issues in the in vivo tissue engineering of three dimensional constructs. The chamber creates a space in the mouse groin by enclosing the epigastric pedicle (artery and vein) with a length of silicon tube [6]. This microenvironment promotes the sprouting of new capillaries from the enclosed pedicle, which supports the survival of implanted cells [7]. This technique is termed intrinsic vascularization [8].

For liver tissue engineering, hepatocytes may appear a logical cell source. However, their limited availability, low proliferative capacity and poor maintenance in culture with dedifferentiation into an immature progenitor-like state [9] and subsequent cell detachment and apoptosis [10] are major issues. Liver progenitor cells (LPCs) are an ideal alternative as they readily proliferate, are maintained robustly in culture and cryostorage, and differentiate 
into bile duct cells (cholangiocytes) and/or hepatocytes [11]. Additionally, LPCs are resistant to hypoxia and ischemic stress [12], which are common in vivo conditions after transplantation. These characteristics indicate that better therapeutic outcomes may be achieved using relatively fewer LPCs than might be required with hepatocytes.

Individual cells in suspension are the most commonly used method of cell-delivery in regenerative medicine. However, spheroid culture has recently been used as an in vivo celldelivery vehicle in order to increase the survival [13] and function [14] of cells when compared to the implantation of cell suspensions derived from monolayer culture. These spheroid cell-delivery studies build on in vitro evidence demonstrating increased cell survival, proliferation and differentiation when cells are cultured as spheroids [15]. This is attributed to increased cell-cell and cell-matrix interactions and restoration of cell polarity, which is diminished in traditional monolayer culture. Despite such encouraging findings, the widespread adoption of spheroid cell-delivery for in vivo studies is hampered by the lack of a reliable method of generating uniform spheroids of known and consistent characteristics, together with a method that allows easy application in an in vivo implantation protocol. Notably, studies exploring the use of hepatocyte spheroids are still rare $[5,16]$, and to our knowledge non-existent with LPC spheroids.

This study reports the increased efficacy of implanting LPCs as spheroids compared to conventional single cell suspensions. An in vivo liver tissue engineering model was established by implanting standardized LPC spheroids of known cell number into a tissue engineering chamber in mice, with analysis of survival and differentiation outcomes. 


\section{MATERIALS \& METHODS}

\subsection{Cell culture}

Bipotential murine oval liver (BMOL) liver progenitor cell (LPC) lines derived from adult mouse liver [17] were cultured at $37^{\circ} \mathrm{C}$ with $5 \% \mathrm{CO}_{2}$, in Williams Media E (Invitrogen, Mulgrave, Victoria, Australia) supplemented with antibiotic-antimycotic, $10 \%$ fetal calf serum, insulin-like growth factor-II (30 ng/mL, R\&D Systems, Minneapolis, USA), epidermal growth factor (50 ng/mL, BD Biosciences, New Jersey, USA), and insulin (10 $\mu \mathrm{g} / \mathrm{mL}$, Sigma-Aldrich, St Louis, Missouri, USA).

\subsection{In vitro spheroid formation}

Cultured LPCs were washed with phosphate-buffered saline (PBS), trypsinized, and counted. After re-suspension in $20 \%$ methylcellulose (Sigma-Aldrich) in supplemented Williams Media E, 20,000 cells in 10-20 $\mu \mathrm{L}$ of media were seeded into each well of MICROTEST ${ }^{\mathrm{TM}} 96$ well U-bottom plates (BD Biosciences). A loose cell aggregate formed by 4 hours, and $50 \mu \mathrm{L}$ of $20 \%$ methylcellulose media was added. At 24 hours, each well was filled to a total volume of $200 \mu \mathrm{L}$ of media, with replacement of $150 \mu \mathrm{L}$ every 24 hours to maintain spheroids. Spheroid formation was observed and photographed via an inverted Olympus IX81 microscope (Olympus Corporation, Tokyo, Japan) (Figure 1B).

Whilst developing this method, preliminary experiments with different cell numbers $(5000$, 10,000 and 20,000 cells) demonstrated that 20,000 cells per well most reliably formed uniform spheroids (shown in supplementary material) in $200 \mu \mathrm{L}$ of methylcellulose media. 


\subsection{In vitro spheroid histology/immunohistochemistry}

Spheroids were examined at 2, 5 or 10 days post formation. After washing in PBS and fixation for 30 minutes in $4 \%$ paraformaldehyde, spheroids were transferred into Biopsy Tissue-Tek ${ }^{\circledR}$ Cryomolds ${ }^{\circledR}$ (ProSciTech, Queensland, Australia) that were filled with 1.5\% agarose at $70^{\circ} \mathrm{C}$ and set. Agarose blocks were then processed and embedded in paraffin, with $5 \mu \mathrm{m}$ longitudinal construct sections cut and mounted on silanized slides.

Immunolabelling was performed with antibodies for Ki67 (1:300 dilution; Lab Vision, California, USA, Catalogue number RM-9106-SO), cleaved caspase 3 (1:150; Cell Signalling, Massachusetts, USA, 9664), pan-cytokeratin (panCK) (1:2000; Dako, California, USA, Z0622), cytokeratin 18 (CK18) (1:50; Abcam, Cambridge, UK, ab32118), hepatocyte nuclear factor 4-alpha (HNF4 $\alpha)$ (1:100; LSBio, Washington, USA, LS-B969), $\beta$-catenin (1:100; Cell Signalling, D10A8), pan-laminin (1:25; Sigma-Aldrich, L9393), fibronectin (1:1000; SigmaAldrich, F3648), and e-cadherin (1:200; Abcam, ab53033). Detailed immunohistochemistry protocols are outlined in the supplementary material.

\subsection{Spheroid dissociation for cell counting}

Spheroids at 1, 2, 5, 7 and 10 days were washed twice with PBS, and then dissociated with $0.25 \%$ trypsin-EDTA (Invitrogen) in PBS. Cell culture plates were agitated on a vibrating platform, with trituration (mechanical pipetting) to ensure dispersal of cell clusters into individual cells. Trypsin-EDTA was neutralized with growth media, and a cell count performed. Four spheroids were dissociated collectively for every cell count, and an average cell number per spheroid obtained. Four separate counts were performed. Trypan blue exclusion was used to estimate the proportion of live cells. 


\subsection{Animals and experimental groups for in vivo implantation}

All animal procedures were performed with the approval of the St Vincent's Hospital Animal Ethics Committee according to National Health and Medical Research Council of Australia animal welfare guidelines. Male SCID mice (ARC, Perth, Western Australia, Australia) aged 6-8 weeks and weighing 20-30 grams were given ad libitum access to chow and water, under a 12 hour day/night cycle. Chloral hydrate was administered as a general anesthetic $(0.4 \mathrm{mg} / \mathrm{g}$ body weight) intra-peritoneally, with a gas mask supplying isofluorene and oxygen.

Bilateral vascularized chambers were created in the groin of every animal (Figure 1A). The right side was seeded with a single cell suspension, and the left with spheroids. The same total number of cells $(200,000$ cells $)$ was seeded in both chambers. Day 2 spheroids were used for all implantations, as in vitro characterization determined this to be the optimal age in culture for implantation. Cells used for single cell suspension and spheroid implantations were at a passage number of less than 5. Chambers were harvested at day 14 and day 45 postimplantation ( $\mathrm{n}=7$ each timepoint).

\subsection{Surgical creation of the vascularized tissue-engineering chamber and LPC implantation}

The microsurgical creation of tissue engineering chambers was performed as described previously [6]. Briefly, with the animal under general anesthesia, the superficial epigastric vascular pedicle branching from the femoral vessels in each groin was surgically exposed. A 5mm length of cylindrical silicon tubing (Dow Corning Corporation, Michigan, USA) split along its longitudinal axis was fitted around this pedicle. The proximal femoral end of the chamber and the split longitudinal seam were sealed with melted bone wax (Ethicon, New Jersey, USA). For every animal, the right chamber was filled with a single cell suspension of 
200,000 cells, and the left chamber with 9 spheroids (200,000 cells in total), both suspended in $50 \mu \mathrm{L}$ of growth factor-reduced Matrigel ${ }^{\mathrm{TM}}$ (BD Biosciences) (Figure 1A). The distal end of the chamber was sealed with bone wax, skin wounds closed with metal clips and the mouse resuscitated.

\subsection{Chamber tissue harvest}

Mice were anesthetized, the implantation site surgically exposed, and the patency of the epigastric pedicle assessed before ligating the pedicle at both ends of the chamber. Tissue constructs (vascular pedicle and surrounding Matrigel tissue contents) were removed from the chamber (Figure 4A), and contruct weight, and volume [18] measured. Fixation in 4\% PFA for 24 hours was followed by routine histological paraffin processing.

\subsection{In vivo construct morphology and morphometry}

Five micron thick longitudinal sections of tissue constructs were immunolabelled with Ki67, panCK, CK18, HNF4 $\alpha, \beta$-catenin (as per in vitro), F4/80 (1:400 dilution; Abcam, ab6640), and CD31 (1:200; BD Biosciences, 553370) as described in supplementary material.

Morphometric analysis of the number of positive-staining cells per $\mathrm{mm}^{2}$ on adjacent panCK and CK18 labelled sections used an automated, systematic random sampling point-counting system (CAST system) (Olympus, Albertslund, Denmark). The observer (KKY) was blinded to the section identity.

The number of panCK + cells per $\mathrm{mm}^{2}$ was determined by systematically sampling $5 \%$ of each section at 40x objective magnification. Similarly, the number of CK18+ cells per $\mathrm{mm}^{2}$ was determined, but because CK18+ cell numbers were considerably less, $15 \%$ of each 
section was sampled, to ensure this population was adequately counted. The total area of construct tissue in each section was measured. The number of panCK + cells or $\mathrm{CK} 18+$ in each field was counted in accordance with the forbidden line rule (18). From the number of cells counted from all fields, the total number of cells per section was calculated by multiplying $100 / 5$ for panCK or $100 / 15$ for CK18. By dividing this number of cells with the area of the section $\left(\right.$ in $\mathrm{mm}^{2}$ ), the number of panCK + or $\mathrm{CK} 18+$ cells $/ \mathrm{mm}^{2}$ was obtained.

Percentage vascular volume was calculated by point-counting $20 \%$ of CD31-labelled sections at 20x magnification, (100-350 points counted/construct). The total number of points counted, and the number of points over a blood vessel wall or lumen was recorded. Percentage vascular volume was calculated using the formula: number of points corresponding to a blood vessel x 100/total number of points counted.

\subsection{Statistical analyses}

Data are expressed as mean \pm SEM. Weights and volumes of day 45 harvested tissue constructs were analysed only using a non-parametric Exact Wilcoxon Mann Whitney test (StatXact v4.0, Cytel Corporation, Massachusetts, USA). For morphometry, the number of panCK + and $\mathrm{CK} 18+$ cells $/ \mathrm{mm}^{2}$ and percentage vascular volume (CD31+ vessels) was analyzed using an independent samples t-test (IBM SPSS Statistics v20.0, IBM, New York, USA). A value of $p<0.05$ was considered significant. 


\section{RESULTS}

\subsection{In vitro characterization of spheroids}

LPCs aggregated into a single cluster within 4 hours, and formed a single uniform spheroid per well within $18-20$ hours. This was close to $100 \%$ efficient, with no formation of secondary cell clusters within the well. Over time, a dense core (confirmed by immunohistochemistry to be apoptotic) was evident, accompanied with an increase in surrounding detached dead cells and cell debris (Figure 1B). Spheroid diameter was approximately $600 \mu \mathrm{m}$ at day 1 and 2 .

Spheroids universally expressed the LPC marker pan-cytokeratin (panCK) (Figure 2A) as well as cytokeratin 18 (CK18) (typically expressed in hepatocytes) (Figure 2B). Ongoing hepatic differentiation was demonstrated by the homogenous and strong immunolabelling for HNF4 $\alpha$ both at day 2 (Figure 2C) and day 10 (not shown). E-cadherin outlined all cell-cell junctions, confirming the epithelial phenotype of LPCs and cell adhesions required to maintain spheroid structure (Figure 2D). $\beta$-catenin increased from day 2 to day 10 (Figures $\mathbf{2 E , F ) , ~ b u t ~ t h e ~ s t a i n i n g ~ p a t t e r n ~ w a s ~ s i m i l a r ~ a t ~ b o t h ~ t i m e - p o i n t s . ~ C y t o p l a s m i c ~} \beta$-catenin was found in the periphery of spheroids, with plasma membrane localization in cells positioned towards the centre. No nuclear staining was observed.

Basement membrane deposition was investigated by pan-laminin and fibronectin immunolabeling. Laminin was found to be diffusely granular throughout spheroids at day 2 (Figure 2G), becoming fibrillar between cells mainly in the periphery of spheroids by day 10 (Figure 2H). Fibronectin was found in greater amounts at both day 2 (Figure 2I) and day 10 (Figure 2J), but as granular deposits in the cytoplasm and cord-like structures in between cells distributed throughout the spheroid. 


\subsection{Optimizing spheroid 'age' for in vivo implantation}

Determining the optimal spheroid 'age' in culture for in vivo implantation was based on two factors. First, spheroids must have compacted enough to remain structurally stable even with pipetting and washing for subsequent in vivo studies. Spheroids were stable from day 2 onwards. Second, spheroids should exhibit maximal proliferation with minimal apoptosis, demonstrated by Ki67 and cleaved caspase 3 immunolabeling respectively. Proliferating cells $(\mathrm{Ki67+})$ were mainly found in the periphery of spheroids, and decreased over time (Figure 3A). In contrast, apoptotic cells (caspase+) were in the central region and increased over time (Figure 3A). Based on these parameters, day 2 spheroids were deemed optimal for implantation.

Dissociation experiments quantified the growth of spheroid cell numbers over time. Importantly, spheroid cell number at day 2 was determined to ensure that the number of cells seeded collectively as spheroids in vivo was equivalent to single cell suspensions. From $20,532 \pm 1,501$ cells per spheroid (mean \pm SEM) at 24 hours to $29,000 \pm 104$ cells at 10 days, spheroid cell numbers gradually increased by $41 \%$ over its initial cell number in 10 days (Figure 3B). At day 2, each spheroid contained $22,407 \pm 645$ cells. More than $\sim 95 \%$ of counted cells were found to be viable by trypan blue exclusion.

\subsection{In vivo implantations: construct harvest, weight and volume}

All animals survived the duration of the in vivo study, with no complications such as infected wounds or displacement of chambers. Active bleeding from all dissected pedicles during harvest indicated patency. Attempts were made to measure the weights and volumes of chamber contents from all groups. However day 14 single cell suspension-implanted tissue 
constructs consisted largely of fragile semi-solid Matrigel (Figure 4B), and could not be weighed. At day 45, spheroid constructs were significantly greater in weight $(p<0.02)$ and volume $(p<0.01)$ than single cell suspension constructs (Figure 4C, D).

\subsection{Construct histology}

Histology demonstrated similar structural components in all groups. The epigastric artery and vein traversed longitudinally along one side of the construct, with multiple capillaries sprouting into the surrounding tissue. A connective tissue capsule with dense inflammatory infiltrate surrounded a central region filled with Matrigel remnants, loose connective tissue, vascular structures, and scattered inflammatory cells, including macrophages (Figure 5).

\subsection{LPC survival and differentiation in spheroid and single cell suspension implantations}

Implanted cells were identified as pan-cytokeratin (panCK) positive or cytokeratin 18 (CK18) positive. Across all groups more panCK + LPCs than CK18+ hepatocyte-like cells were present.

For single cell suspension implantations at day 14 , both LPCs (panCK+) and hepatocyte-like cells $(\mathrm{CK} 18+)$ cells were found in small areas of isolated or loosely clustered cells, with no particular organization or localization. By day 45 the number of LPCs (panCK + cells $/ \mathrm{mm}^{2}$ ) decreased indicating reduced LPC survival over time, although the population of differentiating cells $\left(\mathrm{CK} 18+\right.$ cells $\left./ \mathrm{mm}^{2}\right)$ remained similar to the earlier time-point (Figure 5 $A$ and 7 G, H). 
Implanted spheroids were generally obvious in day 14 constructs as aggregates of cells which were both panCK $+/ \mathrm{CK} 18+$ (Figure 5B, 6A, B). Small groups of migrating LPCs (panCK + ) and differentiating hepatocytes (CK18+) were also scattered throughout the Matrigel region at much greater frequency than with single cell suspension implantations. Spheroid remnants at day 14 were also found to weakly express HNF4 $\alpha$ (Figure 6C), although no $\beta$-catenin staining was evident in these cells (data not shown). By day 45, in two constructs spheroid remnants appeared more loosely aggregated with cells dispersing into the surrounding tissue. These had similar panCK+/CK18+ co-expression as spheroids seen at day 14 . In the remaining five constructs, instead of spheroid remnants, panCK identified small irregular cell clusters which were distributed throughout the chamber (Figures 5B, 7A). CK18+ cells were located with the same distribution, but instead of irregular clusters they displayed hepatic glandular organization into plates and acini-like structures (Figure 7B). Importantly, these structures were strongly positive for HNF4a (Figure 7C), and whilst absent in some structures, $\beta$-catenin was clearly evident in others with either a cytoplasmic (Figure 7D) or membranous localization (Figure 7E). No nuclear $\beta$-catenin was observed.

Numbers of both LPCs and differentiated cells increased over time in spheroid constructs, with morphometric analysis demonstrating a trend towards increased cell survival (panCK+ cells, $p=0.38$, mean \pm SEM 2.4 fold increase) and significantly increased differentiation (CK18+ cells, $p<0.002,5.1$ fold increase) in spheroid-seeded chambers compared to cell suspension at day 14. By day 45, both measures were significantly increased (panCK, $p<0.006,16$ fold increase) (CK18, $p<0.019,6$ fold increase) (Figure 7 G, H).

PanCK + cells had spindle-shaped epithelioid morphology, whereas CK18+ cells were cuboidal, more closely resembling hepatocytes. However no binucleate or polynuclear cells, 
characteristic of the terminal stages of hepatic differentiation, were observed. When considering the difference in cell morphology as well as organization of panCK + and $\mathrm{CK} 18+$ cells, this suggests two divergent populations of cells, where the panCK+ LPCs formed irregular clusters and the CK18+ cells were organizing into tissue-like structures undergoing hepatic differentiation. No obvious biliary ductal structures were observed. Ki67+ proliferative cells were occasionally present in spheroid remnants at day 14 (Figure 6D), as well as hepatic plates and acini at day 45 (Figure 7F), indicating potential for further cell expansion.

\subsection{Construct vascularization}

Intense vascular sprouting from the epigastric pedicle with further vascular remodelling over time was evident, consistent with previous observations [19]. At day 14 extensive CD31+ capillary sprouts were distributed throughout the construct (Figure 8A), with arterioles and venules branching from the main epigastric pedicle. Many blood vessels were perfused with red blood cells. Extravasated blood at the outer margins of the construct suggested presence of immature hyperpermeable capillaries in this region. At day 14 and 45, where spheroid remnants were present blood vessels penetrated throughout the cellular aggregates thus bringing LPCs in contact with the blood circulation (Figure 8B). Interestingly, F4/80+ macrophages were found around and within the core of spheroids (Figure 6D) in close association with LPCs and blood vessels, possibly indicating the recapitulation of an LPC niche.

Extravascular blood was rarely seen at day 45 as the capillary network appeared fully formed (Figure 8C). Morphometric analysis demonstrated a decrease in vascularization over time from day 14 to day 45 (Figure 8D), suggesting some vessel remodelling and possibly 
regression within the vascular network. There was no difference in percentage vascular volume between the two implantation methods (day 14, $p<0.683$ and day $45, p<0.626$ ), suggesting little influence of implantation method on vascularization.

\section{DISCUSSION}

Spheroid cell-delivery holds promise as a method of optimizing cell survival in a range of cell therapies. This study established a protocol for producing consistently uniform spheroids of LPCs, and determined that 2 day culture is optimal for generating spheroids for in vivo implantation. By implanting LPC spheroids of known cell number in an in vivo mouse tissue engineering chamber, significantly enhanced LPC survival and differentiation was achieved compared to single cell implantations of the same cell number.

A critical part of the study design was the implementation of an effective spheroid-forming technique. Many spheroid formation methods exist, including culture in Matrigel $[5,20]$, rocked suspension [21], hanging drop [22], or the use of bioreactors [23]. These are often poorly suited for cell-delivery due to slow spheroid formation, considerable variations in size and shape and hence cell number per spheroid, or difficulties in accessing individual spheroids for manipulation. A simple method to generate standardized spheroids of known cell number is crucial so that the number of cells implanted as spheroids can be controlled, allowing comparison with single cell suspensions. This also facilitates the investigation of the effects of various cell seeding densities. The methylcellulose technique produced uniform spheroids of well-defined architecture. However, they were of a diameter greater than the diffusion limit of oxygen $(100-200 \mu \mathrm{m})$ [24], causing central apoptosis at later time-points in culture. This is a common finding with larger spheroids where hypoxia and inefficient 
nutrient and waste exchange in the core lead to cell death [25]. However, smaller spheroids risk having insufficient cell numbers to allow for cell interaction to form a physiological unit, and their size makes manipulation for in vivo implantations technically challenging.

The co-expression of panCK and CK18 remained unchanged over time in vitro, suggesting that LPCs maintain their bipotential capacity in culture as spheroids. However, the strong in vitro expression of $\mathrm{HNF} 4 \alpha$, a central transcription factor involved in the upstream regulation of major hepatic functions [26], as well as the $\beta$-catenin staining pattern suggests the presence of ongoing hepatic differentiation. $\beta$-catenin, a crucial component of the Wnt pathway involved in liver morphogenesis and differentiation, has been found in the nucleus and cytoplasm of immature cells, with progressive plasma membrane localization as cells differentiate into hepatocytes [27]. The cytoplasmic staining in the periphery of spheroids, and plasma membrane localization in central cells indicates that cells in the core of spheroids are differentiating. Conversely, cells in the periphery are relatively immature, but do not have nuclear staining which would be indicative of a more primitive status.

The investigation of basement membrane $(\mathrm{BM})$ production in spheroids supports these findings. $\mathrm{BM}$ is essential in the development of cell polarity and function, and cell-matrix interactions enhance cell survival as well as govern proliferation and differentiation. Proliferative LPCs are closely associated with a laminin-rich BM niche in the regenerating liver, [28] whereas fibronectin is the main BM component in the adult liver [29]. Greater deposition of laminin was seen in the periphery of spheroids, coincident with the area of proliferation (Ki67+) and relative immaturity (cytoplasmic $\beta$-catenin). In contrast, fibronectin was widespread and more robustly deposited, although not as structurally organized as laminin. This points to the relative immaturity of BM composition, although this was 
progressively developing. Collectively, these findings suggest that LPCs are still at an early to intermediate stage of differentiation in spheroid culture.

Upon in vivo implantation, the penetration of spheroid structures by blood vessels sprouting from the pedicle demonstrates the potential of the chamber in providing a well vascularized implantation environment. Being based on a vascular pedicle with a superficial location, the chamber-engineered tissue may be re-transplanted near the native liver site, and can also be easily removed should any adverse event occur. Additionally its extra-hepatic location is away from a diseased liver environment such as cirrhosis, which may inhibit LPC differentiation as well as contribute to the dedifferentiation of hepatocytes [30].

Morphometric analysis showed significantly increased LPC survival and differentiation in spheroid implantations compared to cell suspension implantations. Importantly, the formation of acini and plate-like structures demonstrated hepatic morphogenesis, with the expression of HNF4 $\alpha$ and membrane-localized $\beta$-catenin in these structures indicating the activation of major pathways involved in hepatic differentiation. Whilst the intermediate loss of $\beta$-catenin in spheroid remnants at day 14 despite its presence both in vitro and more so at day 45 was unexpected, this is possibly analogous to the transient drop in $\beta$-catenin activity during the intermediate stages of embryonic liver development [31].

It is evident that a larger population of surviving cells as well as further differentiation is required to create a sizeable mass of liver tissue. However, the cell survival in spheroid constructs is encouraging, as only 200,000 cells were implanted. Others have demonstrated enhanced cell survival with spheroid cell-delivery compared to single cell suspensions in an in vivo model of myocardial infarction, but this benefit was not maintained over 4 weeks as 
cell survival ultimately diminished [14]. Given this, evidence of sustained cell expansion at the levels apparent in this study despite the seeding density is compelling, and underlines the synergy between the proliferative capacity of LPC spheroids and the vascularized chamber environment. It is also noteworthy that this study was performed in a non-disease model, which lacks regenerative stimuli known to enhance the engraftment of implanted LPCs [20, 32].

In considering the therapeutic application of this model, the newly formed extra-hepatic liver tissue is particularly suitable for correcting metabolic or genetic liver disease, where often only a small but significant portion of liver function is lacking, with the host's native liver relatively intact. Recently it has been suggested that poorly sustained survival of cells transplanted into the liver is due to replacement of transplanted cells by newly differentiated hepatocytes as part of normal cell turnover [33]. In such a case, the extra-hepatic approach described here is ideal in preventing transplanted cells from being replaced by the host's diseased cells.

\section{CONCLUSION}

This study has developed an in vivo liver tissue engineering model based on LPC spheroid implantation that significantly increases cell survival, as well as hepatic morphogenesis and differentiation compared to standard cell-delivery as a single cell suspension. Further studies investigating the functional capacity of newly formed tissue, as well as upscaling by increased seeding density of spheroids may develop this model into a viable alternative for the treatment of liver disease. 


\section{Acknowledgements}

The authors would like to thank Sue McKay, Liliana Pepe, Anna Deftereos, and Amanda Rixon from the Experimental Medical and Surgical Unit at St Vincent's Hospital Melbourne for surgical assistance.

Financial Support:

This work was funded by the St Vincent's Hospital Melbourne Research Endowment Fund, the National Health and Medical Research Council of Australia (Project Grant 1023187), and the Victorian State Government's Department of Innovation, Industry and Regional Development's Operational Infrastructure Support Program. 


\section{REFERENCES}

[1] Dhawan A, Puppi J, Hughes RD, Mitry RR. Human hepatocyte transplantation: current experience and future challenges. Nat Rev Gastroenterol Hepatol. 2010;7(5):288-98. [2] Joseph B, Malhi H, Bhargava KK, Palestro CJ, McCuskey RS, Gupta S. Kupffer cells participate in early clearance of syngeneic hepatocytes transplanted in the rat liver. Gastroenterology. 2002;123(5):1677-85.

[3] Han B, Lu Y, Meng B, Qu B. Cellular loss after allogenic hepatocyte transplantation. Transplantation. 2009;87(1):1-5.

[4] Ohashi K, Waugh JM, Dake MD, Yokoyama T, Kuge H, Nakajima Y, et al. Liver tissue engineering at extrahepatic sites in mice as a potential new therapy for genetic liver diseases. Hepatology. 2005;41(1):132-40.

[5] Navarro-Alvarez N, Soto-Gutierrez A, Chen Y, Caballero-Corbalan J, Hassan W, Kobayashi S, et al. Intramuscular transplantation of engineered hepatic tissue constructs corrects acute and chronic liver failure in mice. J Hepatol. 2010;52(2):211-9.

[6] Cronin KJ, Messina A, Knight KR, Cooper-White JJ, Stevens GW, Penington AJ, et al. New murine model of spontaneous autologous tissue engineering, combining an arteriovenous pedicle with matrix materials. Plast Reconstr Surg. 2004;113(1):260-9.

[7] Tilkorn DJ, Davies EM, Keramidaris E, Dingle AM, Gerrand YW, Taylor CJ, et al. The in vitro preconditioning of myoblasts to enhance subsequent survival in an in vivo tissue engineering chamber model. Biomaterials. 2012;33(15):3868-79.

[8] Lokmic Z, Mitchell GM. Engineering the microcirculation. Tissue Eng Part B Rev. 2008;14(1):87-103. 
[9] Chen Y, Wong PP, Sjeklocha L, Steer CJ, Sahin MB. Mature hepatocytes exhibit unexpected plasticity by direct dedifferentiation into liver progenitor cells in culture. Hepatology. 2012;55(2):563-74.

[10] Vinken M, Decrock E, Doktorova T, Ramboer E, De Vuyst E, Vanhaecke T, et al. Characterization of spontaneous cell death in monolayer cultures of primary hepatocytes. Arch Toxicol. 2011;85(12):1589-96.

[11] Dan YY, Yeoh GC. Liver stem cells: a scientific and clinical perspective. J Gastroenterol Hepatol. 2008;23(5):687-98.

[12] Stachelscheid H, Urbaniak T, Ring A, Spengler B, Gerlach JC, Zeilinger K. Isolation and characterization of adult human liver progenitors from ischemic liver tissue derived from therapeutic hepatectomies. Tissue Eng Part A. 2009;15(7):1633-43.

[13] Bhang SH, Cho SW, La WG, Lee TJ, Yang HS, Sun AY, et al. Angiogenesis in ischemic tissue produced by spheroid grafting of human adipose-derived stromal cells. Biomaterials. 2011;32(11):2734-47.

[14] Lee WY, Wei HJ, Lin WW, Yeh YC, Hwang SM, Wang JJ, et al. Enhancement of cell retention and functional benefits in myocardial infarction using human amniotic-fluid stem-cell bodies enriched with endogenous ECM. Biomaterials. 2011;32(24):5558-67.

[15] Pampaloni F, Reynaud EG, Stelzer EH. The third dimension bridges the gap between cell culture and live tissue. Nat Rev Mol Cell Biol. 2007;8(10):839-45.

[16] Ota K, Saito S, Fujisawa K, Tanaka N. Xenotransplantation of spheroidal aggregatecultured hepatocytes. Transplant Proc. 1997;29(1-2):912-3.

[17] Tirnitz-Parker JE, Tonkin JN, Knight B, Olynyk JK, Yeoh GC. Isolation, culture and immortalisation of hepatic oval cells from adult mice fed a choline-deficient, ethioninesupplemented diet. Int J Biochem Cell Biol. 2007;39(12):2226-39. 
[18] Howard CV, Reed MG. Unbiased stereology. 2nd ed: Oxford: BIOS Scientific Publishers; 2005.

[19] Rophael JA, Craft RO, Palmer JA, Hussey AJ, Thomas GP, Morrison WA, et al. Angiogenic growth factor synergism in a murine tissue engineering model of angiogenesis and adipogenesis. Am J Pathol. 2007;171(6):2048-57.

[20] Forster N, Palmer JA, Yeoh G, Ong WC, Mitchell GM, Slavin J, et al. Expansion and hepatocytic differentiation of liver progenitor cells in vivo using a vascularized tissue engineering chamber in mice. Tissue Eng Part C Methods. 2011;17(3):359-66.

[21] Brophy CM, Luebke-Wheeler JL, Amiot BP, Khan H, Remmel RP, Rinaldo P, et al. Rat hepatocyte spheroids formed by rocked technique maintain differentiated hepatocyte gene expression and function. Hepatology. 2009;49(2):578-86.

[22] Chinzei R, Tanaka Y, Shimizu-Saito K, Hara Y, Kakinuma S, Watanabe M, et al. Embryoid-body cells derived from a mouse embryonic stem cell line show differentiation into functional hepatocytes. Hepatology. 2002;36(1):22-9.

[23] Tostoes RM, Leite SB, Serra M, Jensen J, Bjorquist P, Carrondo MJ, et al. Human liver cell spheroids in extended perfusion bioreactor culture for repeated-dose drug testing. Hepatology. 2012;55(4):1227-36.

[24] Helmlinger G, Yuan F, Dellian M, Jain RK. Interstitial pH and pO2 gradients in solid tumors in vivo: high-resolution measurements reveal a lack of correlation. Nat Med. 1997;3(2):177-82.

[25] Lin RZ, Chang HY. Recent advances in three-dimensional multicellular spheroid culture for biomedical research. Biotechnol J. 2008;3(9-10):1172-84.

[26] Si-Tayeb K, Lemaigre FP, Duncan SA. Organogenesis and development of the liver. Developmental cell. 2010;18(2):175-89. 
[27] Monga SP, Micsenyi A, Germinaro M, Apte U, Bell A. beta-Catenin regulation during matrigel-induced rat hepatocyte differentiation. Cell Tissue Res. 2006;323(1):71-9.

[28] Kallis YN, Robson AJ, Fallowfield JA, Thomas HC, Alison MR, Wright NA, et al. Remodelling of extracellular matrix is a requirement for the hepatic progenitor cell response. Gut. 2011;60(4):525-33.

[29] Zhu C, Coombe DR, Zheng MH, Yeoh GC, Li L. Liver progenitor cell interactions with the extracellular matrix. J Tissue Eng Regen Med. 2012.

[30] Rountree CB, Mishra L, Willenbring H. Stem cells in liver diseases and cancer: recent advances on the path to new therapies. Hepatology. 2012;55(1):298-306.

[31] Micsenyi A, Tan X, Sneddon T, Luo JH, Michalopoulos GK, Monga SP. Beta-catenin is temporally regulated during normal liver development. Gastroenterology. 2004;126(4):1134-46.

[32] Turner RA, Wauthier E, Lozoya O, McClelland R, Bowsher JE, Barbier C, et al. Successful transplantation of human hepatic stem cells with restricted localization to liver using hyaluronan grafts. Hepatology. 2012 Sep 19; doi: 10.1002/hep.26065. [Epub ahead of print].

[33] Furuyama K, Kawaguchi Y, Akiyama H, Horiguchi M, Kodama S, Kuhara T, et al. Continuous cell supply from a Sox9-expressing progenitor zone in adult liver, exocrine pancreas and intestine. Nat Genet. 2011;43(1):34-41. 


\section{FIGURE LEGENDS}

Figure 1. Experimental design. (A) An in vivo animal model was established by surgically creating bilateral chambers, and implanting 200,000 dissociated cells as a single cell suspension into the right chamber, and 9 spheroids (200,000 cells in total) into the left chamber. Two time-points post-implantation were studied, day 14 and day 45 ( $\mathrm{n}=7$ per time point). (B) In vitro microscopy demonstrating spheroids in culture. A single, uniform spheroid/well formed that was stable for 10 days. Note the increase in dead cells and cell debris surrounding the spheroid over time. From in vitro characterization, day 2 spheroids were found to be optimal for use in chamber implantations.

Figure 2. In vitro characterization of liver progenitor cell spheroids (representative histology). At day 2, spheroids stained homogenously positive for (A) pan-cytokeratin (panCK), and (B) Cytokeratin 18 (CK18), indicative of their bipotential capacity. (C) Hepatocyte nuclear factor 4-alpha (HNF4a) demonstrated activation of hepatic differentiation pathways, and (D) E-cadherin indicated the epithelial phenotype of LPCs as well as cell adhesion required to maintain the integrity of the spheroid. Similar homogenous staining was seen up to day 10 (data not shown). (E) $\beta$-catenin, a key component of the Wnt pathway that regulates cell proliferation and differentiation in the liver, was found at day 2 to be mainly cytoplasmic in the periphery of spheroids (arrowhead) suggestive of relatively immature, proliferative cells in this region. Towards the centre of the spheroids plasma membrane localization (arrow) was evident, demonstrating a staining pattern seen in more mature hepatocytes. (F) At day 10 increased $\beta$-catenin activity was present, although the same pattern of cytoplasmic staining in the peripheral cells and plasma membrane localization in central cells was seen. (G) Laminin, a basement membrane component, was diffuse throughout the spheroid at day 2 both between cells and within the cytoplasm. (H) However, by day 10 laminin was seen as fibrillar material between cells (arrows) mostly in the periphery, suggestive of organization of this basement membrane component into a more mature, functional structure. Fibronectin, a major component of basement membranes in the mature liver, was found in large amounts in spheroids at both day 2 (I) and day 10 (J). Whilst some cord-like deposition in between cells was evident throughout the spheroid, much of the fibronectin was also within the cytoplasm of cells often as granular deposits. This suggests robust production of this basement membrane component, but a relative lack of organization into a mature basement membrane as seen with laminin.

Figure 3. Selection of optimal time-point of spheroid development for use in implantations. (A) Ki67+ proliferative cells (arrowheads) were found mainly in the periphery of spheroids, and decreased over 10 days. Cleaved caspase 3+ apoptotic cells (arrowheads) were mainly located in the central region, and increased over 
time. (B) Spheroid dissociation over 10 days ( $n=16$ per time-point). Cell number per spheroid gradually increased from $20,532 \pm 1501$ cells per spheroid at 24 hours (mean $\pm S E M$ ) to $29,000 \pm 1104$ cells per spheroid at 10 days, representing a $41 \%$ increase in cell number over this time period. At day 2 , when spheroids were deemed optimal for implantation, each spheroid contained $22,407 \pm 645$ cells. More than $\sim 95 \%$ of counted cells were found to be viable by trypan blue exclusion.

Figure 4. Harvest of chambers. (A) Removal of the silicon chamber yielded a three-dimensional tissue construct, from a day 14 spheroid-seeded chamber construct shown here (10x magnification). The epigastric pedicle is prominent (large arrow), with multiple capillaries sprouting into the adjacent tissue (small arrows). (B) For weight and volume measurement, single cell suspension constructs at day 14 were composed mainly of Matrigel and too fragile for this procedure, although the epigastric pedicle is prominent here also (large arrow) (7x magnification). (C) Weight and (D) Volume measurements demonstrated a significant increase in day 45 spheroid constructs compared to single cell suspensions. ( ${ }^{*}$ denoting $p<0.02$ in weight and ${ }^{* *}$ for $p<0.01$ in volume measurements, non-parametric Exact Wilcoxon Mann Whitney test).

Figure 5. General overview of harvested chamber constructs. All chamber constructs were immunolabelled using panCK and CK18 on serial sections. General structural components of chamber constructs are labelled: central Matrigel region $(m)$, vascular pedicle $(p)$, connective tissue capsule (c). (A) In single cell suspension constructs at both day 14 and day 45 , very few panCK + or CK18+ cells were present (small patch of cells indicated by arrow). (B) In spheroid chambers, at day 14 spheroid remnants were clearly labelled by both panCK and CK18 (arrows). By day 45 both panCK+ and CK18+ cells (arrows) were found to be dispersed throughout the Matrigel region $(m)$ surrounding the epigastric pedicle $(p)$, enclosed within the connective tissue capsule (c) of the construct.

Figure 6. Representative immunolabelling in serial sections of spheroids from day 14 constructs Spheroid remnants were evident in day 14 constructs (arrows, inset figures). They were positive for (A) LPC marker pancytokeratin (panCK), but also weakly positive for (B) hepatocyte marker cytokeratin $18(\mathrm{CK} 18)$. CK18 ${ }^{+}$cells in the periphery of the spheroid remnant and in the surrounding tissue demonstrated organization into acini and hepatic plate-like formations (arrowheads). (C) The presence of HNF4a activation in cells within spheroid remnants suggests ongoing differentiation. (D) Ki67 immunolabelled proliferating cells were seen ocassionally in spheroid remnants (arrowheads). (E) F4/80 immunolabelled macrophages penetrated into the core of a spheroid, suggesting cell-cell interaction between LPCs, macrophages, and possibly blood vessels.

Figure 7. Spheroid constructs at day 45. (A) PanCK+ cells were seen in irregular clusters between blood vessels, inflammatory and connective tissue cells in the central Matrigel region. (B) CK18+ cells in the Matrigel 
region demonstrated glandular organization of cells into hepatic acini with a lumen often evident. (C) Hepatic acini-like structures were strongly positive for HNF4a, indicating hepatic differentiation. Some, but not all of these acini and plate structures were positive for $\beta$-catenin, either demonstrating (D) cytoplasmic $\beta$-catenin (arrowheads) indicative of relatively immature cells, or (E) plasma membrane localization (arrowheads) more typically seen in mature hepatocytes. Collectively this suggests organization of implanted cells into hepatic structures with varying levels of differentiation. (F) A small number of cells within distinct hepatic acini were found to be proliferative and stained positive for Ki67 (arrows). (G) Morphometric analysis demonstrated increased panCK + cells in spheroid constructs at day $14(p<0.38$, independent samples t-test, 2.4 fold increase) compared to single cell suspension implantations. This difference was significant at day $45\left({ }^{* *}, p<0.006,16\right.$ fold increase). (H) Similarly, significantly increased CK18+ cells numbers were found in spheroid constructs compared to single cell suspension implantations at day $14\left({ }^{* *}, p<0.002,5.1\right.$ fold increase) and day $45\left({ }^{*}, p<0.019,6\right.$ fold increase).

Figure 8. Vascularization in tissue engineering constructs (CD31 immunolabelling). (A) At day 14, large dilated vessels (arrow) indicated immature vascular formation (spheroid construct shown, with similar observations in single cell suspension constructs). (B) Where spheroid remnants were present at day 14, blood vessels were found to surround (large arrow) as well as penetrate (small arrows) into the spheroid core to vascularize the cell aggregates. (C) By day 45 , construct blood vessels were more mature than at 14 days with a smaller diameter (arrows). (D) There was no difference between the two implantation methods in terms of percentage vascular volume (day $14, p<0.683$ and day $45, p<0.626$ ) suggesting little influence of implantation method on vascularization. However, vascularization was noted to decrease with both implantation methods from day 14 to day 45, in line with the observation that with vascular remodelling, vessels became less dilated and decrease in number. 


\section{University Library}

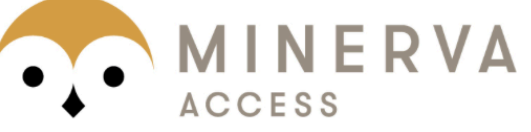

A gateway to Melbourne's research publications

Minerva Access is the Institutional Repository of The University of Melbourne

Author/s:

Yap, KK;Dingle, AM;Palmer, JA;Dhillon, RS;Lokmic, Z;Penington, AJ;Yeoh, GC;Morrison, WA;Mitchell, GM

Title:

Enhanced liver progenitor cell survival and differentiation in vivo by spheroid implantation in a vascularized tissue engineering chamber

Date:

2013-05-01

Citation:

Yap, K. K., Dingle, A. M., Palmer, J. A., Dhillon, R. S., Lokmic, Z., Penington, A. J., Yeoh, G. C., Morrison, W. A. \& Mitchell, G. M. (2013). Enhanced liver progenitor cell survival and differentiation in vivo by spheroid implantation in a vascularized tissue engineering chamber. BIOMATERIALS, 34 (16), pp.3992-4001. https://doi.org/10.1016/j.biomaterials.2013.02.011.

Persistent Link:

http://hdl.handle.net/11343/43800 